\title{
Nonlinear Partial Differential Equations Arising from Prescribed Curvature Problems
}

\author{
Man Chun Leung \\ National University of Singapore, 21 Lower Kent Ridge Road, Singapore \\ E-mail: matlmc@nus.edu.sg
}

\begin{abstract}
We consider two prominent nonlinear partial differential equations (nonlinear PDE) linked to the prescribed curvature problems, namely, the Minkowski problem and the Kazdan-Warner/Nirenberg problem (prescribed scalar curvature problem). This article addresses some of the modern techniques in analysis used to draw out a number of the profound features in these equations.
\end{abstract}

Keywords: blow-up, Monge-Ampére Equation, optimal transport problem

\section{Introduction}

In 1827 , Gauss ${ }^{[1]}$ presents to the Royal Society of Gottingen his study on surfaces, and introduces Gaussian curvature. Some seventy six years later, Minkowski publishes an article ${ }^{[2]}$ on the prescribed Gaussian curvature problem (the famed Minkowski problem). It asks: Given a strictly positive smooth function $G$ defined on $S^{2}$, can we find a strictly convex compact surface $\sum \subset I R^{3}$ such that the Gauss curvature of $\sum$ at the point $x \in \sum$ equals $G(\mathbf{n}(x))$ ? Here $\mathbf{n}(x)$ denotes the unit outward normal to $\sum$ at $x$. The prescribed function $G(\mathbf{n}(x))$ is supposed to satisfy the condition

$$
\int_{S^{2}} \mathcal{G}(\mathbf{n}) \mathbf{n} d S_{\mathbf{n}}=\overrightarrow{0}
$$

This can be naturally generalized to hypersurfaces in $I R^{n}$. We refer to $\S 1$ a for the rather standard notations we use in this article.

Arising from this prescribed curvature problem is the Monge-Ampére equation of the type

$$
\operatorname{Det}[\operatorname{Hess}(u)]=g(\nabla u)
$$

Eugenio Calabi states in [3], "From the geometric view point it (the Minkowski problem) is the Rosetta Stone, from which several related problems can be solved." Classic as it is, in recent years there are major breakthroughs on MongeAmpere type equations, together with its relation to the optimal transport problem [cf. $\$ 2 \mathrm{~g}]$.

Concerning the square matrix $\operatorname{Hess}(u)$, there are two important quantities associated to it, namely, the determinant and the trace (that is, the Laplacian of $u, \Delta u$ ). The next prescribed curvature problem we consider here is indeed related to $\Delta u$. Known as the KazdanWarner/Nirenberg problem ${ }^{[4]}{ }^{[5]}$, it asks: Given a smooth function $K$ defined on $S^{n}(n \geq 3)$ can we find a conformal deformation of the standard metric $g_{1}$ on $S^{n}$, so that its scalar curvature equals to $K$ ? Arising from this prescribed scalar curvature problem is the semi-linear partial differential equation of the type

$$
\Delta_{1} U-\left[\tilde{c}_{n} n(n-1)\right] U+\left[\tilde{c}_{n} K\right] U^{\frac{n+2}{n-2}}=0 \text { in } S^{n}(U>0, n \geq 3)
$$

Here $K$ is supposed to fulfill the Kazdan-Warner condition ${ }^{[6]}$

$$
\int_{S^{n}} X(K) U^{\frac{2 n}{n-2}} d S=0\left[\text { where } X(K)=X \cdot \nabla_{1} K\right]
$$

where $X$ is any conformal Killing vector field (one that generates a family of conformal transformations). 
In this expository article, our aim is to present a global overview on some of the interesting recent developments in these two equations, focusing on the "beauty" behind the apparent simple problems. The interested readers may also like to consult [7-26] for additional information on the topics. In $\$ 2$, we discuss the weak (Alexandrov) solution to certain Monge-Ampére equations and the Minkowski problem. The links to the optimal transport problem are also discussed. §3 is devoted to the prescribed scalar curvature problem, in particular when the prescribed function exhibits certain symmetries. The Lyapunov-Schmidt (finite dimension) reduction method is introduced in $\S 4$. And in $\S 5$ we discuss the construction of various types of blow-up sequence of solutions of the conformal scalar curvature equation.

$\S 1 \mathrm{a}$. General conditions, assumptions and conventions. Throughout this work,

$$
S^{n}=\left\{x=\left(x_{1}, \ldots, x_{n+1}\right) \in I R^{n+1} \mid x_{1}^{2}+\cdots+x_{n+1}^{2}=1\right\} \quad(\mathrm{n} \geq 2),
$$

equipped with the induced metric $g_{1}$. Let us denote by $\Delta_{1}$ the Laplace-Beltrami operator associated with $g_{1}$ on $S^{n}$, and $\left|S^{n}\right|$ the volume of $\left(S^{n}, g_{1}\right)$. Denote by $B_{x}(\rho)$ the open (geodesic) ball in $\left(S^{n}, g_{1}\right)$ with center at $x \in S^{n}$ and radius $\rho \in(0, \pi)$.

$\bullet_{1}$ Likewise, $\Delta$ is the Laplace-Beltrami operator associated with Euclidean metric $g_{o}$ on $I R^{n}$. Moreover, the norm \|\| and the inner product $\langle$,$\rangle are defined via g_{o}$. Denote by $B_{y}(r)$ the open ball in $\left(I R^{n}, g_{o}\right)$ with center at $y$ and radius $\left.r\right\rangle$ 0 , and $\partial B_{y}(r)$ its boundary. Whenever there is no risk of misunderstanding, we suppress $d y$ from the integral expressions on domains in $\mathrm{IR}^{n}$.

${ }_{2}$ We use "Det" to denote the determinant of a square matrix, and "Hess" to indicate the Hessian of a $C^{2}$ function defined on $I R^{n}$. Denote by $J_{T}$ the Jacobian of $T$, where $T$ is $C^{1}$ map $T: O \rightarrow I R^{n}$, defined on an open set $O \subset I R^{n}$.

$\bullet_{3}$ In this article, $\tilde{c}_{n}=\frac{n-2}{4(n-1)}$, where $n \geq 3$. We observe the practice on using ' $C$ ', possibly with sub-indices, to denote various positive constants, which may be rendered differently from line to line according to the contents.

\section{The Minkowski problem}

Based on his earlier works on the Minkowski problem ${ }^{[27]}{ }^{[28]}$, Alexandrov ${ }^{[29]}$ introduces a notion of generalized (weak) solution to the Monge-Ampére equation (known nowadays as the Alexandrov solution). It may be of interest to note that one of Alexandrov's doctoral students is Grigori Perelman. $§ 2 \mathrm{a}$. Convex function and its subdifferential. Let $\Omega \subset I R^{n}$ be a convex domain, and $u: \Omega \rightarrow I R$ a convex function. It is known that $u$ is uniformly Lipschitz in every compact subset in $\Omega$, and differentiable almost everywhere. The subdifferential of $u$ at a point $x_{o} \in \Omega$ is given by the collection

$$
\partial u\left(x_{o}\right):=\left\{\vec{p} \in I R^{n} \mid u(x) \geq u\left(x_{o}\right)+\vec{p} \cdot\left(x-x_{o}\right) \text { for all } x \in \Omega\right\}
$$

Here $\vec{p}$ is taken to be at the default position. We first observe that, when $u \in C^{1}(\Omega)$, the tangent (hyper) plane of the hypersurface was defined by the graph

$$
\left\{(x, u(x))=\left(x_{1}, \cdots x_{n}, u\left(x_{o}\right)\right) \in I R^{n+1} \mid x \in \Omega\right\}
$$

at $x_{o} \in \Omega$ is given by

$$
x_{n+1}=u\left(x_{o}\right)+\left[\nabla u\left(x_{o}\right)\right] \cdot\left(x-x_{o}\right)
$$

Thus,

$\partial u\left(x_{o}\right)$ is equal to $\nabla u\left(x_{o}\right)$ when $u \in C^{1}(\Omega)$.

Let us compare $u$ with another convex function $v$ defined in $\Omega$. If $O \subset \Omega$ is an open and bounded set such that $u \leq v$ in $O$, and $u=v$ on $\partial O$

then

$$
\partial v(O) \subset \partial u(O),
$$


This leads to the Alexandrov's maximum principle (see Theorem (10) in [17]).

$\S 2 \mathrm{~b}$. Measure (Monge-Ampére). For every Borel set $E \subset \Omega$, define the Monge-Ampére measure as

$$
\mu_{u}(E)=\lambda *\left(\bigcup_{x \in E} \partial u(x)\right)
$$

Here we use the Lebesgue measure $\lambda *(\bullet)$. For a convex function $u$, we have the following:

$-\mu_{u}$ is a Borel measure.

$\bullet_{2}$ In case $u \in C^{2}(\Omega)$, then

$$
\mu_{u}=[\operatorname{Det} \operatorname{Hess}(u)] d x .
$$

- 3 The Monge-Ampére measure is "stable" under uniform convergence (see Proposition 2.4 in [17]).

$\S 2 \mathrm{c}$. Weak (Alexandrov) solution. As in the above, $\Omega \subset I R^{n}$ is an open bounded convex set. Let

$$
f: \Omega \times I R \times I R^{n} \rightarrow I R^{+} .
$$

be a positive function. A convex function $u: \Omega \rightarrow R$ is said to be an Alexandrov solution to the Monge-Ampére equation

$$
\operatorname{Det}[\operatorname{Hess}(u)]=f(x, u, \nabla u) \text { in } \Omega
$$

if

$\mu_{u}=f(x, u, \nabla u) d x$ in the sense of Borel measures,

that is $\mu_{u}(E)=\int_{E} f(x, u, \nabla u) \mathrm{dx}$ for all Borel measurable set $E \subset \Omega$.

$\S 2$ d. Convergence. A great advantage of working on weak solutions is the following convergence (stability) theorem. Let $u_{k}: \Omega \rightarrow$ IR be a sequence of convex Alexandrov solutions of

$\mu_{u_{k}}=v_{k}$ in $\Omega, u_{k}=0$ on $\partial \Omega$.

Here $v_{k}$ is a sequence of nonnegative Borel measures with $\sup _{k} v_{k}(\Omega)<\infty$. Moreover,

$v_{k} \rightarrow^{*} v$ weakly to a Borel measure $v$.

Then $\left\{u_{k}\right\}$ converges (locally uniformly) to $u_{\infty}$, which is convex and an Alexandrov solution of

$$
\mu_{u_{\infty}}=v \text { in } \Omega, u_{\infty}=0 \text { on } \partial \Omega \text {. }
$$

This allows us to solve a simpler problem first, and take the limit, obtaining a (weak) solution. See [17] for more information.

$\S 2 \mathrm{e}$. Boundary value problem. Suppose that $v_{o}$ is a nonnegative Borel measure with $v_{o}(\Omega)<\infty$. By using a sequence of finite sum of atomic measures, which converge weakly to $v_{o}$, and a Perron-type argument, Alexandrov ${ }^{[3]}$ shows that there exists a unique convex function $u: \Omega \rightarrow I R$ such that

$$
\mu_{u}=v_{o} \text { in } \Omega, u=0 \text { on } \partial \Omega .
$$

With stronger conditions: assuming that $\Omega$ is uniformly convex of class $C^{3}, v_{o}=f d y$, where $f \in C^{2}(\bar{\Omega})$, and

$$
0<\lambda \leq f \leq \frac{1}{\lambda} \text {. }
$$

Pogorelov ${ }^{[30]}$ shows the existence (and uniqueness) of a $C^{2, a}(\bar{\Omega})$ solution to the Dirichelt problem (10) via the renowned continuity method.

As for the (weak) Alexandrov solution, Caffarelli ${ }^{[31]}$ obtains regularity results, showing that strictly convex 
Alexandrov solutions of (10) are locally $C_{\text {loc }}^{1, \alpha}(\Omega)$ [condition (11) required]. Refer also to [32].

$\S 2$ f. Weak solution to the Minkowski problem. For an open bounded convex domain $K \subset \operatorname{IR}^{n+1}$ with $0 \in K$, let the boundary $\partial K$ be given by

$\partial K=\left\{\rho(x) x \mid x \in S^{n}, \rho: S^{n} \rightarrow I R^{+}\right\}$.

Similar to the subdifferential, for $z=\rho(x) x \in \partial K$, define

$$
N_{K}(z)=\left\{n \in S^{n} \mid K \subset\left\{w \in I R^{n+1} \mid\langle n,(w-z)\rangle \leq 0\right\}\right\}
$$

The Gaussian curvature measure is defined by

$\mu_{K}(E)=\left\|N_{K}(E)\right\|_{H^{n}}$ for all Borel measurable set $E \subset S^{n}$.

Here $\|\bullet\|_{H^{n}}$ is the $n$-dimensional Hausdorff measure on $S^{n}$. As in the case of the MongeAmpére measure, $\mu_{K}$ a Borel measure.

The Minkowski problem can be recast into this form: Given a Borel measure $v$ on $S^{n}$, can we find an open bounded convex set $K$ containing the origin so that

$$
\mu_{K}=v ?
$$

Alexandrov ${ }^{[1][13]}$ shows that the necessary and suffcient conditions for the existence of a weak solution to equation (13) are [see for example [33] for the complete statement and more details ] $v\left(S^{n}\right)=\left|S^{n}\right|$ and $v(F)<\left|S^{n}\right|-\left|F^{*}\right|$ for all Borel measurable set $F \subset S^{n}$.

Here $F^{*}$ is the dual angle defined via

$$
F^{*}=\left\{y \in S^{n} \mid y \cdot x \leq 0 \text { for all } x \in F\right\}
$$

Suppose that a portion of $\partial K$ can be parameterized as the graph of a convex $C^{2}$-function $u: \mathcal{O} \rightarrow$ IR, then the Gaussian curvature of the hypersurface defined by the graph of $u$ is given by

In the above, $\mathcal{O} \in I R^{n}$ is an open set. With $v=f(x) d H^{n}$, equation (13) can be expressed locally in the form

$\operatorname{Det}[\operatorname{Hess}(u)]=f \cdot\left(1+\|\nabla u\|^{2}\right)^{\frac{n}{2}+1}$.

One can then apply the regularity theory developed by Caffarelli to draw the conclusion that $\partial K$ is strictly convex and of class $C^{1, \gamma}$. Moreover, if $f \in C^{0, \alpha}$, then $u \in C^{2, \alpha}$. Conditions on $f$ apply-refer to [24].

$\S 2 \mathrm{~g}$. The optimal transport problem. The problem was formalized by the French mathematician Gaspard Monge in 1781 [34]. In modern day language, let $X, Y \subset I R^{n}$ be open bounded domains, with probability measures $\mu=f d x$ (on $X$ ) and $v=h d y$ (on $Y$ ), respectively $\left(f, h>0\right.$ ). A $C^{1}$-diffeomorphism $T: X \rightarrow Y$ is called a transport map with respect to the pair $(f, h)$ if the following Jacobian equation is satisfied:

$f(x)=h(T(x)) \cdot \operatorname{Det} J_{T} \quad$ for all $x \in X$.

It follows from (15) that

Det $J_{T}=\frac{f(x)}{h(T(x))}>0$ and $\int_{X} f(x) d x=\int_{Y} h(y) d y$.

Elsewhere, (15) is interpreted in a weak sense:

$\int_{Y} \psi(y) d v(y)=\int_{X} \psi(T(x)) d \mu(x)$ for all Borel function $\psi: Y \rightarrow I R$.

A transport map $T_{o p}$ is a solution to the optimal transport problem [ from $(X, f)$ to $\left.(Y, h)\right]$ with quadratic cost if $T_{o p}$ 
minimizes the integral [among all the transport maps described in (15) ]

$$
\int_{x}\|x-T(x)\|^{2} f(x) d x
$$

By considering the Monge-Ampére equations and Helmholtz-type decompositions, Brenier ${ }^{[35]}$ obtains the following existence and uniqueness result: There exists a lower semicontinuous convex function $u: I R^{n} \rightarrow I R \cup\{+\infty\}$ such that

$$
T_{o p}=\nabla u \quad(\mu-a . e .)
$$

and

$$
\operatorname{Det}[\operatorname{Hess}(u)]=\frac{f}{h \circ \nabla u} \quad(\mu-\text { a.e. })
$$

Note that in dimension three, if $u$ is $C^{2}$, then the vector field $\nabla u$ is curl free (intuitively, "rotational free"; rotation can be seen as a waste in most transport).

$\S 2 \mathrm{~h}$. General cost function and the Ma-Trudinger-Wang (MTW) tensor. Consider general cost function

$$
c: X \times Y \rightarrow \mathrm{IR}^{+} \cup\{0\},
$$

which is of class $C^{4}\left(\|c\|_{C^{4}(X \times Y)}<\infty\right)$, together with the injectivity of the maps

$$
y \mapsto-D_{x} c(x, y) \text { and } x \mapsto-D_{y} c(x, y)
$$

and

$$
\operatorname{Det}\left(D_{x y} c\right)(x, y) \neq 0 \quad \text { for all }(x, y) \in X \times Y \text {. }
$$

With these, one can define the notion of $c$-convexity, and $c$-subdifferential. Similar result as in (19) can be obtained. See [17] for more details. With regard to regularity of the weak solution, Ma-Trudinger-Wang ${ }^{[36]}$ finds a fourthorder tensor, with which the positive definiteness is a suffcient condition for the smoothness of the optimal transport solution. Loeper sheds light on the geometric interpretation of the MTW condition, linking it to the connectedness of the $c$-subdifferential.

\section{Conformal scalar curvature problem}

In this section and the following two, we treat the dimension $\mathrm{n} \geq 3$. The prescribed scalar curvature problem in $S^{n}$ asks for a positive solution $U$ of the nonlinear partial differential equation

$$
\Delta_{1} U-\tilde{c}_{n} n(n-1) U+\left(\tilde{c}_{n} K\right) U^{\frac{n+2}{n-2}}=0 \text { in } S^{n}(\mathrm{U}>0),
$$

where $K$ is a prescribed function on $S^{n}$. A stand-out feature of equation (19) is the exponent $\frac{n+2}{n-2}$. Indeed, it is related to the critical Sobolev embedding: the injection $H^{1,2}\left(S^{n}\right) \rightarrow L^{\frac{2 n}{n-2}}\left(S^{n}\right)$ is not compact, typified by blow-up gathering at critical point(s) of $K$. When $K$ equals to a positive constant, say, $K \equiv 4 n(n-1)$, equation (19) has a family of positive solutions (via the stereographic projection $\dot{P}$ which sends the north pole $N$ to $\infty$ ):

$$
\left\{U_{\lambda, \xi}(x):=\left(\frac{\lambda}{\lambda^{2}+\|y-\xi\|^{2}}\right)^{\frac{n-2}{2}} \cdot\left(\frac{1+\|y\|^{2}}{2}\right)^{\frac{n-2}{2}} \text { with }(\lambda, \xi) \in I R^{+} \times I R^{n}\right\}
$$

Here $y=\dot{P}(x)$ for $x \in S^{n} \backslash N$, and the value at the north pole is given by $U_{\lambda, \xi}(N):=[\lambda / 2]^{\frac{n-2}{2}}$. In particular, any sequence $\left\{U \lambda_{i}, \xi_{i}\right\}_{i=1}^{\infty}$ with $\lambda_{i} \rightarrow 0^{+}$and $\xi_{i} \rightarrow \overrightarrow{0}$ is a blow-up sequence (blow-up point at the south pole $S$ ).

Broadly speaking, there are two approaches to the problem: avoiding the blow-up (via blow-up analysis), or making use of it (for example, the Lyapunov-Schmidt reduction method). The following result provides a good picture on the first 
(i) $K$ is a smooth Morse function [namely, all its critical points (collected in the set denoted by Crt) are nondegenerate].

(ii) $\Delta_{1} K\left(x_{c}\right) \neq 0$ for all $x_{c} \in$ Crt.

(iii) $\sum_{x_{x} \in \mathrm{Crrt}_{c}}(-1)^{\operatorname{lnd}\left(K, x_{c}\right)} \neq(-1)^{n}$, here $\mathrm{Crt}_{<}=\left\{x_{c} \in \mathrm{Crt} \mid \Delta_{1} K\left(x_{c}\right)<0\right\}$.

(iv) $K$ is "suffciently" close to a positive constant. Then equation (20) has a positive solution (precisely, see Theorem 7.1 in [37], pp. 103). Recall that the index of a non-degenerate critical point is the number of negative eigenvalues of the Hessian matrix at that point.

We observe that in case $\mathrm{Crt}_{<}$contains only one point, say at the north pole $N$, then it must be the peak (maximal point), and hence (together with the non-degenerate condition)

$$
\operatorname{Ind}(K, N)=n \Rightarrow \sum_{x_{c} \in C r_{<}}(-1)^{\operatorname{Ind}\left(K, x_{c}\right)}=(-1)^{\operatorname{Ind}(K, N)}=(-1)^{n} .
$$

Moreover, via the Kazdan-Warner condition (4), if $K$ is strictly decreasing from $N$ to $S$, measured via the geodesics, then equation (20) does not have any positive solution at all (cf. also [39]).

$\S 3 \mathrm{a}$. The flow method. Chen and $\mathrm{Xu}^{[15]}$ study the scalar curvature flow equation, and gain a better understanding on the point " $K$ is "suffciently" close to a positive constant". Consider the parabolic equation

$$
\frac{\partial U}{\partial t}(t, x)=\frac{n-2}{4} \cdot\left[\alpha(t) \cdot K(x)-R_{g U(t, \bullet)}(x)\right] U(t, x) \quad U(0, x)=U_{o}(x)
$$

for $(x, t) \in I R^{+} \times S^{n}$. Here

$$
R_{g U(t, \bullet)}(x)=-\frac{1}{\tilde{c}_{n}} \cdot \frac{\Delta_{1} U(t, x)-\tilde{c}_{n} n(n-1) U(t, x)}{[U(t, x)]^{\frac{n+2}{n-2}}}
$$

is the scalar curvature of the conformal metric $g_{U(t, \bullet)}=\left[U(t, \bullet]^{\frac{4}{n-2}} \cdot g_{1}\right.$, and the zoom factor

$$
\alpha(t):=\left[\int_{S^{n}} R_{g U(t, \bullet)} \cdot\left[U(t, \bullet)^{\frac{2 n}{n-2}} d V_{g_{1}}\right] /\left[\int_{S^{n}} K \cdot[U(t, \bullet)]\right]^{\frac{2 n}{n-2}} d V_{g_{1}}\right]
$$

is included to keep the volume of $\left(S^{n}, g_{U(t, .)}\right)$ constant along the flow (see [15]).

It is known that [15] either

$$
\|U(t, \bullet)\|_{H^{2, p}} \text { for all } t \in(0, \infty),
$$

or there is a finite collection of points ("blow-up points) $\left\{b_{1}, \cdots, b_{L}\right\} \subset S^{n}(L \geq 1)$ so that (modulo a subsequence)

$\left\{U_{i}\right\}$ is uniformly bounded in any compact subsets of $S^{n} \backslash\left\{b_{1}, \cdots, b_{L}\right\}$,

and

$$
\liminf _{i \rightarrow \infty}\left(\frac{1}{\left|S^{n}\right|} \int B_{b j(\rho)}\left|R_{i}\right|^{\frac{n}{2}} d V_{g_{i}}\right)^{\frac{2}{n}} \geq n(n-1) \quad[1 \leq \mathrm{j} \leq \mathrm{L}] \quad \text { for any } \rho>0 .
$$

In case there is only one blow-up point, say at $Q \in S^{n}$, then we know the followings ${ }^{[15]}$.

$\cdot{ }_{1} Q$ is a critical point of $K$ [ that is, $\nabla_{1} K(Q)=0$ ].

$\bullet_{2} \Delta_{1} K(Q) \leq 0$.

-3 $\lim _{i \rightarrow \infty} Q_{K}\left[u_{i}\right]=\frac{n(n-1)}{[K(Q)]^{\frac{n-2}{n}}}$.

Using the infinite dimension Morse Theory, Chen and Xu show that, under the conditions in (i)-(iii) [see [15] for a 
more general statement], for $n \geq 5$, if

$$
\max _{S^{n}} K / \min _{S^{n}} K<2^{\frac{2}{n-2}}
$$

then equation (20) has a positive solution.

§3. Symmetry. The flow method combines well with function $K$ which processes the following symmetries.

(29) $K$ is symmetric for a mirror reflection upon a hyperplane $H \subset I R^{n+1}$ ( $H$ passes through the origin). As the situation is invariant under a rotation, we assume (without loss of generality) that $H$ is the hyperplane perpendicular to the $x_{1}$ - axis. In this way, the symmetry is expressed as

$K\left(\gamma_{m}(x)\right)=K(x)$, where $\gamma_{m}: S^{n} \rightarrow S^{n}$ is given by $\gamma_{m}\left(x_{1}, x_{2}, \cdots, x_{n+1}\right)=\left(-x_{1}, x_{2}, \cdots, x_{n+1}\right)$

for $x=\left(x_{1}, \cdots, x_{n+1}\right) \in S^{n}$. As such $F=\left\{\left(0, x_{2}, \cdots, x_{n+1}\right) \in S^{n}\right\}=H \cap S^{n}$ is the fixed point set.

(30) $K$ is invariant under a rotation $\gamma_{\theta}$ of angle $\theta=\pi / k$ (with the axis being a straight line in $I R^{n+1}$ passing through the origin). Here $k>1$ is an integer. Likewise, without loss of generality, we take it that indeed the straight line is the $x_{n+1}$-axis. In this case the fixed point set $F=\{N, S\}$. In [40] we obtain the following result.

Theorem (31). Suppose that $K>0$ is a smooth function on $S^{n}$ which is invariant under the symmetry described in either (29) or (30). Assume that

$$
x_{m} \in F \text { with } K\left(x_{m}\right)=\max _{F} K \Rightarrow \Delta_{1} K\left(x_{m}\right)>0,
$$

and

$$
\left(\max _{S^{n}} K\right)^{\tau}<2 \cdot\left(\min _{F} K\right)^{\tau}
$$

where $\tau=\frac{n-2}{2}$. Then, equation (20) has a smooth positive solution $U$ for $K$.

One of the key element in the proof of (31) is to construct a highly concentrated bubble located at a maximal point in $F$ as the initial condition $U_{o}$ of the flow. See [40]. This brings us to the next topic on making use of the blow-up process via the Lyapunov-Schmidt reduction method to find the solutions of equation (20).

\section{Lyapunov-Schmidt reduction method}

$\S 4$ a. Simple plan. Given Banach spaces $X$ and $Y$, consider a nonlinear functional equation

$$
F(x, \varepsilon)=0 \in(Y), x \in X .
$$

In the above,

$$
F: X \times(-1,1) \rightarrow Y
$$

is $C^{1}$. Suppose we have a solution at $(0,0$,$) , that is,$

$$
F(0,0)=0,
$$

and $F_{x}(0,0)$ being invertible, then the implicit function theorem assures that, for $|\varepsilon|$ small enough, we have a solution $\left(x_{\varepsilon}, \varepsilon\right)$ of equation (35), that is

$F\left(x_{\varepsilon}, \varepsilon\right)=0(|\varepsilon|$ small enough $)$.

On other hand, if

$\operatorname{Ker} F_{x}(0,0)=X_{O} \neq \varnothing$, 
yet $F_{x}(0,0)$ is still an Fredholm operator, with

Range $F_{x}(0,0)=Y_{o}$.

We split $X$ and $Y$ into

$X=X_{o} \oplus X_{c}, Y=Y_{o} \oplus Y_{c}\left(\operatorname{dim} X_{o}<\infty, \operatorname{dim} Y_{c}<\infty\right)$.

Let $P: Y \rightarrow Y_{o}$ be the projection.

Equation (35) is equivalent to

$$
\begin{aligned}
& P \circ F(x, \varepsilon)=0, \\
& (I-P) \circ F(x, \varepsilon)=0 .
\end{aligned}
$$

Maximize the subspace where (36) can be solved. For equation (36), consider finding solutions of the type

$P \circ F\left(x_{o}+x_{c}, \varepsilon\right)=0, x_{o} \in X_{o}$, and $x_{c} \in X_{c}$.

This equation has a solution at

$(0,0,0)$

and its derivative at $(0,0,0)$ (with respect to $\left.x_{c}\right)$ is now invertible. Thus, applying the implicit function theorem on the functional

$P \circ F: X_{o} \oplus X_{c} \oplus I R \rightarrow Y_{o}$,

we obtain a solution

$\left(x_{o}, x_{c}\left(x_{o}, \varepsilon\right), \varepsilon\right)$ for $\varepsilon \approx 0$ and $x_{o} \approx 0$.

By substituting $x_{c}\left(x_{o}, \varepsilon\right)$ into the second equation [that is, (37)], one finds a finite-dimensional equation

$$
(I-P) \circ F\left(x_{o}+x_{c}\left(x_{o}, \varepsilon\right), \varepsilon\right)=0
$$

to be solved by finding $x_{o} \in X_{o}\left(\operatorname{dim} X_{o}<\infty\right)$.

$\S 4$ b. Lyapunov-Schmidt reduction method - the case of one bubble. In order to illustrate how this simple idea works on the scalar curvature equation (20) [with suitable modification according to the situation], we introduce the one bubble case. Consider the stereographic projection

$$
\begin{aligned}
& \dot{P}: S^{n} \backslash\{N\} \rightarrow I R^{n} \\
& x \mapsto y=\dot{P}(x), \text { where } y_{i}=\frac{x_{i}}{1-x_{n+1}}, 1 \leq i \leq n
\end{aligned}
$$

Here $x=\left(x_{1}, \cdots, x_{n+1}\right) \in S^{n} \backslash\{N\} \subset I R^{n+1}$, and $N=(0, \cdots, 0,1)$. The conformal factor is given by

$$
g_{1}(x)=\left[\frac{4}{\left(1+\|y\|^{2}\right)^{2}}\right] g_{o}(y) \text { for } y=\dot{P}(x) \text {. }
$$

Note that the conformal factor is related to the standard bubble [ cf. (40) ]. Set 
$v(y):=U\left(\dot{P}^{-1}(y)\right) \cdot\left(\frac{2}{1+\|y\|^{2}}\right)^{\frac{n-2}{2}}$ and $\check{K}(y):=K\left(\dot{P}^{-1}(y)\right)$ for $y \in I R^{n}$

Suppose that $U$ is a positive $C^{2}$ solution of equation (20). Then $v$ satisfies the equation

$\Delta_{o} v+\left(\tilde{c}_{n} \check{K}\right) v^{\frac{n+2}{n-2}}=0$ in $I R^{n}$.

Note that in this setting

$$
\Delta_{o} V_{\lambda, \xi}+n(n-2) V_{\lambda, \xi}^{\frac{n+2}{n-2}}=0 \text { in } I R^{n},
$$

where

$$
V_{\lambda, \xi}(y)=\left(\frac{\lambda}{\lambda^{2}+\|y-\xi\|^{2}}\right)^{\frac{n-2}{2}} \text { with }(\lambda, \xi) \in I R^{+} \times I R^{n} .
$$

Collect them into

$$
Z:=\left\{V_{\lambda, \xi} \in D^{1,2} \mid(\lambda, \xi) \in I R^{+} \times I R^{n}\right\} .
$$

The functional corresponding to equation (38) is given by

$$
I(f)=\frac{1}{2} \int_{I R^{n}}\langle\nabla f, \nabla f\rangle-\left(\frac{n-2}{2 n}\right) \cdot \int_{I R^{n}}\left(\tilde{c}_{n} \cdot \check{K}\right) f_{+}^{\frac{2 n}{n-2}} \text { for } f \in D^{1,2}
$$

Here $f_{+}$denotes the positive part of $f$. In (42), the Hilbert space is chosen to be

$$
D^{1,2}=D^{1,2}\left(I R^{n}\right):=\left\{f \in L^{\frac{2 n}{n-2}}\left(I R^{n}\right) \cap W_{l o c}^{1,2}\left(I R^{n}\right) \mid \int_{I R^{n}}\langle\nabla f, \nabla f\rangle<\infty\right\},
$$

with the inner product defined by

$$
\langle f, \psi\rangle_{\nabla}:=\int_{I R^{n}}\langle\nabla f, \nabla \psi\rangle \text { for } f, \psi \in D^{1,2}
$$

Thus, we seek a critical point $v$ of the functional (42), that is,

$$
I(v)=0,
$$

which can be expressed as

$$
I^{\prime}(v)[h]=\int_{I R^{n}}\left[\langle\nabla v, \nabla h\rangle-\left(\tilde{c}_{n} \cdot K\right) v_{+}^{\frac{n+2}{n-2}} \cdot h\right]=0 \text { for all } h \in D^{1,2}
$$

Let

$\check{K}=4 n(n-1)+\varepsilon \cdot H$, where $|\varepsilon|$ is small.

Here $H$ is a bounded continuous function on $I R^{n}$. (45) can be rewritten as 


$$
\begin{aligned}
I_{\varepsilon}(f): & =\frac{1}{2} \int_{I R^{n}}\left[\langle\nabla f, \nabla f\rangle-(n-2)^{2} f_{+}^{\frac{2 n}{n-2}}\right]-\varepsilon \cdot\left(\frac{n-2}{2 n}\right) \cdot \int_{I R^{n}}\left(\tilde{c}_{n} H\right) f_{+}^{\frac{2 n}{n-2}} \\
& =I_{o}(f)+\varepsilon \cdot G(f) .
\end{aligned}
$$

Here

$$
I_{o}(f)=\frac{1}{2} \int_{I R^{n}}\left[\langle\nabla f, \nabla f\rangle-(n-2)^{2} f_{+}^{\frac{2 n}{n-2}}\right], \check{K}
$$

and

$$
G(f)=\bar{C}_{-1} \int_{I R^{n}} H f_{+}^{\frac{2 n}{n-2}} \text { for } f \in D^{1,2}, \text { where } \bar{C}_{-1}:=-\frac{(n-2)^{2}}{8 n(n-1)} .
$$

Via the Riesz Representation Theorem [see (52) and (53) below], (47) can be expression as a nonlinear functional equation

$$
F_{S}(v, \varepsilon): D^{1,2} \times(-1,1) \rightarrow \mathrm{D}^{1,2} .
$$

Moreover, via (39) and (46),

$$
F_{S}\left(V_{\lambda, \xi}, 0\right)=0
$$

Consider the derivative of $F_{S}$ at $\left(V_{\lambda, 5}, 0\right)$. It is related to

$$
\left(I_{o}^{\prime \prime}\left(V_{\lambda, \xi}\right)[\phi] h\right)=\int_{I R^{n}}\left[\langle\nabla \phi, \nabla h\rangle-n(n+2) V_{\lambda, \xi}^{\frac{4}{n-2}} \cdot \phi \cdot h\right] \text { for } \phi, h \in D^{1,2}
$$

Thus, the kernel is given by

$$
\left(I_{o}^{\prime \prime}\left(V_{\lambda, \xi}\right)[\phi] h \equiv 0 \text { for all } h \in D^{1,2}\right.
$$

which points us to the equation ( in weak sense)

$$
\Delta_{o} \phi+n(n+2) \cdot\left(V_{\lambda, \xi}^{\frac{4}{n-2}} \cdot \phi\right)=0 \text { in } I R^{n}
$$

Introducing orthogonality. We are interested in finding a solution $\phi \in D^{1,2}$ of the linear inhomogeneous equation

$$
\Delta \phi+n(n+2) \cdot\left(V_{\lambda, \xi}^{\frac{4}{n-2}} \cdot \phi\right)=\left(V_{\lambda, \xi}^{\frac{4}{n-2}} \cdot h\right), \text { where } h \in D^{1,2} .
$$

Note that, multiplying both sides of (50) by

$$
\left(\lambda \cdot \frac{\partial}{\partial \lambda}\right) V_{\lambda, \xi}
$$

and upon integration, we have 


$$
\int_{I R^{n}}\left(V_{\lambda, \xi}^{\frac{4}{n-2}} \cdot h\right) \cdot\left[\left(\lambda \cdot \partial_{\lambda}\right) V_{\lambda, \xi}\right]=\left\langle h,\left[\left(\lambda \cdot \partial_{\lambda}\right] V_{\lambda, \xi}\right\rangle_{\nabla}=0\right.
$$

Here we apply

$$
\Delta V_{\lambda, \xi}+n(n-2) V_{\lambda, \xi}^{\frac{n+2}{n-2}}=0 \Rightarrow \Delta\left(\partial_{\lambda} V_{\lambda, \xi}\right)+n(n+2) \cdot\left[V_{\lambda, \xi}^{\frac{4}{n-2}} \cdot\left(\partial_{\lambda} V_{\lambda, \xi}\right)\right]=0
$$

and the integration by parts formula. Similarly for other derivatives. Hence

$$
\left\langle h,\left[\left(\lambda \cdot \partial_{\lambda}\right) V_{\lambda, \xi}\right]\right\rangle_{\nabla}=\left\langle h,\left[\left(\lambda \cdot \partial \xi_{\left.\right|_{j}}\right) V_{\lambda, \xi}\right]\right\rangle_{\nabla}=0 \text { for } j=1,2, \cdots, n .
$$

Equation (50) can be seen in weak form: the problem is equivalent to finding a $\phi \in D^{1,2}$ such that

$$
\begin{aligned}
& \int_{I R^{n}}\left\{\Delta \phi+n(n+2) \cdot\left(V_{\lambda, \xi}^{\frac{4}{n-2}} \cdot \phi\right)-\left(V_{\lambda, \xi}^{\frac{4}{n-2}} \cdot h\right)\right\} \cdot \psi=0 \text { for all } \psi \in D^{1,2} \\
& \Leftrightarrow \int_{I R^{n}}\langle\phi, \psi\rangle_{\nabla}+\left[-n(n+2) \int_{I R^{n}}\left(V_{\lambda, \xi}^{\frac{4}{n-2}} \cdot \phi\right) \cdot \psi\right]=\left[-\int_{I R^{n}}\left(V_{\lambda, \xi}^{\frac{4}{n-2}} \cdot h\right) \cdot \psi\right] \text { for all } \phi \in D^{1,2} .
\end{aligned}
$$

Here we apply the integration by parts formula. Via the Riesz Representation Theorem, the problem can be cast in the form of finding a solution (in $D^{1,2}$ ) of the (functional) equation

$$
(I d+K)(\phi)=h_{D}
$$

Here $I d$ is the identity map interpreted by

$$
\langle I d(\phi), \psi\rangle_{\nabla}=\langle\phi, \psi\rangle_{\nabla},
$$

and

$$
\langle K(\phi), \psi\rangle_{\nabla}=\left[-n(n+2) \int_{I R^{n}}\left(V_{\lambda, \xi}^{\frac{4}{n-2}} \cdot \phi\right) \cdot \psi\right] \text { for all } \psi \in D^{1,2} .
$$

It can be checked that $K: D^{1,2} \rightarrow D^{1,2}$ is a compact linear operator (via the RellichKondrachov Theorem). [A compact linear operator is a linear operator $L$ from a Banach space $X$ to another Banach space $Y$, such that the image under $L$ of any bounded subset of $X$ is a relatively compact subset of $Y$ (i.e., the closure is compact).]

Similarly, $h_{D} \in D^{1,2}$ is defined by

$$
\left\langle h_{D}, \psi\right\rangle_{\nabla}=\left[-\int_{I R^{n}}\left(V_{\lambda, \xi}^{\frac{4}{n-2}} \cdot h\right) \cdot \psi\right] \text { for all } \psi \in D^{1,2} .
$$

Note that 


$$
\begin{aligned}
& \left|\int_{I R^{n}}\left(V_{\lambda, \xi}^{\frac{4}{n-2}} \cdot h\right) \cdot \psi\right| \\
& \leq\left[\int_{I R^{n}}\left(V_{\lambda, \xi}^{\frac{4}{n-2}} \cdot h\right)^{\frac{2 n}{n+2}}\right]^{\frac{n+2}{2 n}} \cdot\left(\int_{I R^{n}}|\psi|^{\frac{2 n}{n-2}}\right)^{\frac{n-2}{2 n}} \\
& \leq\left[\left(\int_{I R^{n}}\left(V_{\lambda, \xi}^{\frac{4}{n-2}}\right)^{\frac{2 n}{n+2} \cdot \frac{n+2}{4}}\right)^{\frac{4}{n+2}} \cdot\left(\int_{I R^{n}}|h|^{\frac{2 n}{n+2} \cdot \frac{n+2}{n-2}}\right)^{\frac{n-2}{n+2}}\right] \cdot\left(\int_{I R^{n}}|\psi|^{\frac{2 n}{n-2}}\right)^{\frac{n-2}{2 n}} \\
& \leq\left[\int_{I R^{n}}\left(V_{\lambda, \xi}^{\frac{4}{n-2}}\right)^{\frac{n}{2}}\right]^{\frac{2}{n}} \cdot\|h\|_{\nabla} \cdot\|\psi\|_{\nabla} \\
& \leq\left(\int_{I R^{n}} V_{\lambda, \xi}^{\frac{2 n}{n-2}}\right)^{\frac{2}{n}} \cdot\|h\|_{\nabla} \cdot\|\psi\|_{\nabla}=\tilde{C}_{n} \cdot\left|S^{n}\right| \cdot\|h\|_{\nabla} \cdot\|\psi\|_{\nabla} .
\end{aligned}
$$

The form of (52) allows us to use Fredholm alternative method to solve the equation. The kernel $N \subset D^{1,2}$ of $(I d+K)$

is identified with the solution space $N$ of the linear equation

$$
\Delta \phi_{o}+n(n+2) \cdot V_{\lambda, \xi}^{\frac{4}{n-2}} \cdot \phi_{o}=0 \quad\left(\phi_{o} \in D^{1,2}\right)
$$

This can be precisely determined [5]:

$$
N=\operatorname{Span}\left\{\left(\lambda \cdot \partial_{\lambda}\right) V_{\lambda, \xi},\left(\lambda \cdot \partial \xi_{\left.\right|_{1}}\right) V_{\lambda, \xi}, \cdots,\left(\lambda \cdot \partial \xi_{\left.\right|_{n}}\right) V_{\lambda, \xi}\right\} .
$$

Moreover, these $(n+1)$ functions are orthogonal to each other with respect to the inner product $\langle,\rangle_{\nabla}$. See, for examples, [37] [41]. Write

$$
D^{1,2}=N \oplus N_{\perp},
$$

where $N_{\perp}$ is the orthogonal complement of $N$. By the self-adjointness of $(I+K)$,

$(I d+K): N_{\perp} \rightarrow N_{\perp}$ is an isomorphism.

Applying the Fredholm alternative method, equation (52) is solvable if and only if $h_{D} \in N_{\perp}$

Recalling relation (53), we have 
$h_{D} \in N_{\perp} \Leftrightarrow\left\langle h_{D},\left(\lambda \cdot \partial_{\lambda}\right) V_{\lambda, \xi}\right\rangle_{\nabla}=0 \Leftrightarrow\left\langle\left(V_{\lambda, \xi}^{\frac{4}{n-2}} \cdot h\right), \frac{\partial V_{\lambda, \xi}}{\partial \lambda}\right\rangle_{\int}=0 \Leftrightarrow\left\langle h, \frac{\partial V_{\lambda, \xi}}{\partial \lambda}\right\rangle_{\nabla}=0$

Similarly for the other derivatives with respect to the components of $\xi$. Therefore

$h \in N_{\perp}$

In general, for $h \notin N_{\perp}$, write

$h=h_{\perp}+\left[-h_{/ /}\right] \Leftrightarrow h_{\perp}=h+h_{\perp}$,

where

$h_{\perp} \in N_{\perp}$ and $h_{/ /} \in N \Rightarrow h_{/ /}=a_{o} \cdot \lambda \frac{\partial V_{\lambda, \xi}}{\partial \lambda}+\sum_{j=1}^{n} b_{j} \cdot \lambda \frac{\partial V_{\lambda, \xi}}{\partial \xi_{j}}$

(The coeffcients $a_{o}$ and $b_{j}$ are uniquely determined.) The above discussion, together with elliptic regularity theory ${ }^{[42]}$, shows that there is a unique solution $\phi \in N_{\perp}$ of the inhomogeneous equation [after checking the relation in (53)]

$$
\begin{aligned}
& \Delta \phi+n(n+2) \cdot\left(V_{\lambda, \xi}^{\frac{4}{n-2}} \cdot \phi\right)= \\
& \left(V_{\lambda, \xi}^{\frac{4}{n-2}} \cdot h\right)+\left[a_{o} \cdot V_{\lambda, \xi}^{\frac{4}{n-2}} \cdot \lambda \frac{\partial V_{\lambda, \xi}}{\partial \lambda}+\sum_{j=1}^{n} b_{j} \cdot V_{\lambda, \xi}^{\frac{4}{n-2}} \cdot \lambda \frac{\partial V_{\lambda, \xi}}{\partial \xi_{j}}\right]
\end{aligned}
$$

Moreover, the coeffcients $a_{o}$ and $b_{j}$ are also uniquely determined by $h$. We sum up the discussion in the following propositions. See also [43] for more general situation.

Proposition (61). Given $h \in D^{1,2}$, there exists a unique $\phi \in N_{\perp}$ which solves equation (60), where the coeffcients $a_{o}$ and $b_{j}$ are uniquely determined by $h$ via the decompostion (58).

Proposition (62). For any $z \in Z, I_{o}^{\prime \prime}(z)$, as rendered in (48), is an index zero Fredholm operator. Moreover,

$$
\operatorname{KerI}_{o}^{\prime \prime}(z)=N
$$

and

$$
I_{o}^{\prime \prime}(z): N_{\perp} \rightarrow N_{\perp}
$$

is an isomorphism.

The flow chart.

The simplicity of the Lyapunov-Schmidt reduction method is expounded in the book ${ }^{[37]}$. Instead of repeating the discussion in [37], we present the chart on the set-up of the Lyapunov-Schmidt reduction method, and encourage readers to refer to the award winning book ${ }^{[37]}$. 


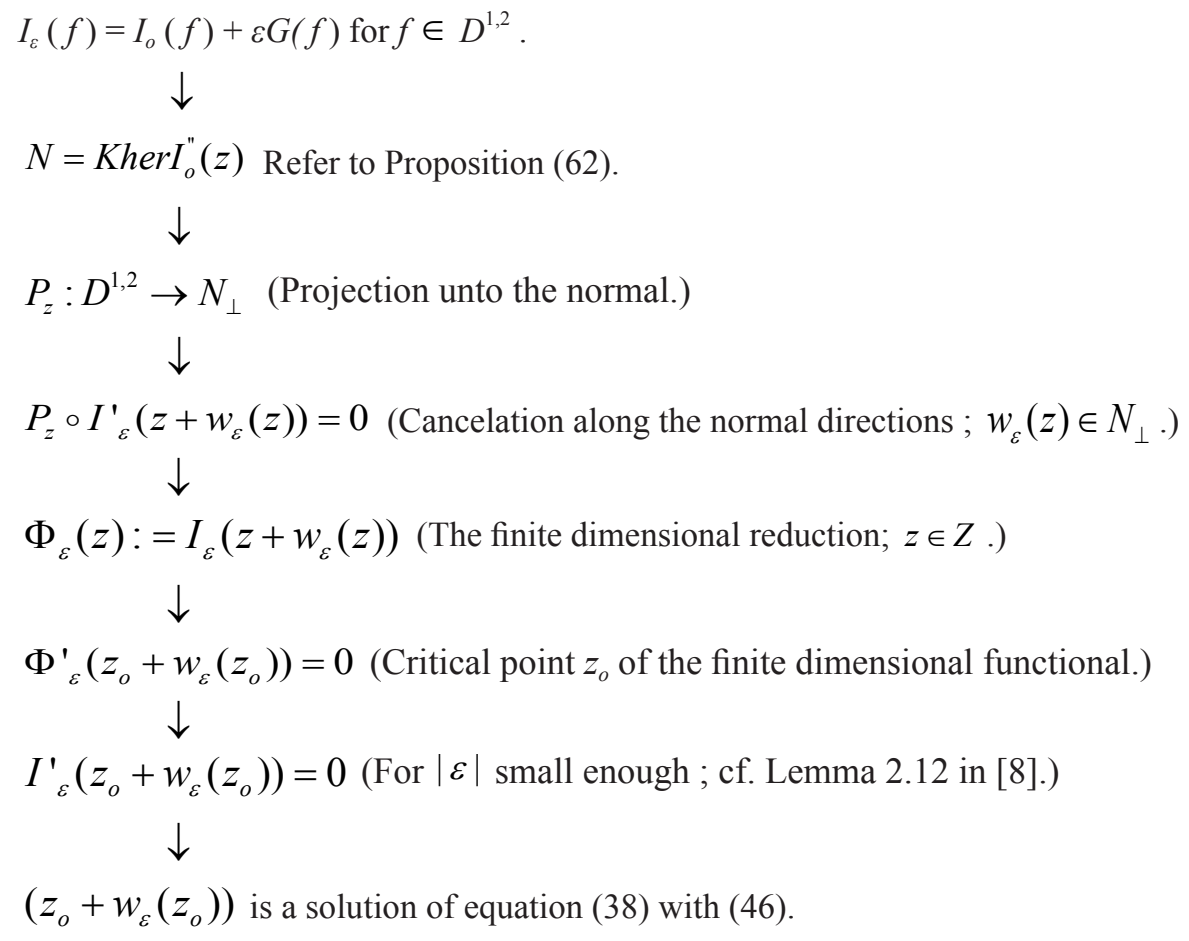

By using Lyapunov-Schmidt reduction method (with one bubble) and a degree counting argument, a version of result as described in §3(i)-(iv) is obtained in [37] (Theorem (63), pp.63).

$\S 4 \mathrm{c}$. The Lyapunov-Schmidt reduction scheme sans perturbation: the case of two or more bubbles. In the above description, the Lyapunov-Schmidt reduction method is considered on those $K$ which is a perturbation of a positive constant [recall (46)]. A new insight is introduced in [43], where Wei and Yan bring home to the point that when a large number of standard bubbles are arranged near the critical points of $K$ (having the same positive value), one can still apply the Lyapunov-Schmidt reduction method, this time without the requirement on $\varepsilon$ in (46) being close to zero. Thus, the number of bubbles replaces the parameter $\varepsilon$.

In [44], the author and Zhou show that by "planting" one bubble each near one of the twin (pseudo-)peaks, the Lyapunov-Schmidt reduction method is also applicable without the need for $K$ being close to a constant. In this case, the gap between the "peaks" takes the place of the parameter $\varepsilon$. In brief, we show that the reduced functional has two main contributions, one from the critical point, and the other one from the interaction with the other bubble (Proposition (35) [44]). By properly balancing these two effects, it can be shown that the prescribed scalar curvature equation (38) has a solution if the "twin peaks" are close enough. Moreover, as the two bubbles are highly concentrated near the twin pseudo-peaks, other critical points (if any) do not contribute to the consideration. This is in harmony with a theme in [45] (cf. also [20]) that concentration can be put to good use to find solutions of equation (20).

\section{Construction of blow-up sequences}

Recent research sheds light on the blow-up process for equation (1). A dividing line is the isolated blow-ups, which include [35]

(i) simple blow-up (intuitively, a single bubble appears) and

(ii) towering blow-up (two or more bubbles are stacked up-roughly speaking).

The more complicated non-isolated blow-ups include

(iii) aggregated blow-up [two or more bubbling sequences converge to the blow-up point, which is (locally) the only blow-up point], and

(iv) cluster blow-up [being a cluster point (or accumulation point) of a sequence of blow-up points].

The elegant Lyapunov-Schmidt reduction method is used by Ambrosetti and Malchiodi ${ }^{[37]}$ to find infinite number of solutions to the Yamabe equation (see [46] [47] [48] for further development). It also allows us to construct simple blowup sequence of positive solutions of equation (1) [41] [37]. Using similar finite dimension reduction method, Wei and Yan consider in their beautiful work ${ }^{[43]}$ the existence of infinite number of solutions, amounting to the case of cluster blow-up. 
In [50], the author and Zhou show that by incorporating two bubbles, the Lyapunov-Schmidt reduction method can also be used to construct an infinite sequence of positive solutions of equation (20) so that the south pole is an aggregated blow-up point, or a towering blow-up point ( $K$ being fixed).

§5a. Delaunay-Fowler solution. The blow-up process is also related to equation (38) with a singularity at the origin. To zoom into the origin, let us introduce the log-cylindrical coordinates $(t, \theta)$, where

$$
t=-\ln \|y\| \text { and } \theta=\frac{y}{\|y\|} \text { for } y \in I R^{n} \backslash\{0\} .
$$

Observe that $\|y\| \rightarrow 0^{+}$as $t \rightarrow \infty$. Allow v be as in equation (38). The function

$$
w(t, \theta):=\left.\|y\|\right|^{\frac{n-2}{2}} \times v(y) \text { for } t \in I R \text { and } \theta \in S^{n-1}
$$

satisfies the equation

$$
\frac{\partial^{2} w}{\partial t^{2}}+\Delta_{\theta} w-\frac{(n-2)^{2}}{4} \cdot w+\left[c_{n} \cdot \tilde{K}\right] w^{\frac{n+2}{n-2}}=0 \text { in } I R \times S^{n-1}
$$

Here $\Delta_{\theta}$ is the Laplacian for the standard unit sphere $S^{n-1}$ in $I R^{n}$, and

$$
\tilde{K}(t, \theta):=\check{K}(y) \text {, with }\|y\|=e^{-t} \text { and } y /\|y\|=\theta \text {. }
$$

Under (62), the standard bubble

$$
\left(\frac{1}{1+\|y\|^{2}}\right)^{\frac{n-2}{2}} \text { is transformed into }\left(\frac{1}{2 \cosh t}\right)^{\frac{n-2}{2}} \text {. }
$$

$\S 5$ b. Radial solutions. We are interested in the radial solutions of equation (63) with $\tilde{K} \equiv 4 n(n-1)$. In this case, (63) becomes the following autonomous O.D.E.

$$
w^{\prime \prime}(t)-\frac{(n-2)^{2}}{4} \cdot w(t)+n(n-2)[w(t)]^{\frac{n+2}{n-2}}=0 \text { in IR. }
$$

For our discussion, equation (64) devises a 'almost perfect' way to stack bubbles.

Positive smooth solutions of (64) are known as Delaunay-Fowler type solutions. By a re-parameterization, we standardize the solutions so that

$$
w(0)=\max _{t \in I R} w(t)
$$

Being periodic ${ }^{[31][33]}$, the Delaunay-Fowler type solutions can be indexed by the neck-size which is given by $\eta:=\inf _{t \in I R} w(t)$. For $\eta=0, w(t)=(2 \cosh t)^{(2-n) / 2}$ with 'infinite period'(cf. [51] [53]). The relation between $\eta$ and the period $T_{d}$ is described in [45].

In our presentation, we find it more convenient to index the Delaunay-Fowler type solutions by the period $T_{d}$. Thus for $T_{d} \gg 1$, denote by $w_{T d}$ the Delaunay-Fowler type solution with period $T_{d}$, neck-size $\eta_{T d}>0$, and with standardization (65). Furthermore, for any fixed number D $>0$,

$$
w_{T_{d}}(t) \rightarrow w_{\infty}(t):=:(2 \cosh t)^{(2-n) / 2} \text { in }[-D, D] \text { as } T_{d} \rightarrow \infty
$$

Geometrically, as noted in [51], the metrics $w_{T_{d}}^{\frac{4}{n-2}}\left(d t^{2}+d \theta^{2}\right)$ converge as $T_{d} \rightarrow \infty$ to a bead of spheres of same radius that are arranged along a fixed axis.

$\S 5$ c. Truncation. One can truncate the Delaunay-Fowler type solution $w_{T_{d}}$ by introducing $\frac{T_{d}}{2}$ 


$$
\bar{w}_{k, T_{d}}(t)= \begin{cases}(2 \cosh t)^{(2-n) / 2} & \text { for } t \leq-1, \text { where } 0<1 \leq \frac{T_{d}}{2}, \\ w_{T_{d}}(t) & \text { for } 1<t<k \cdot T_{d}-1, \text { where } k \in I N, \\ {\left[2 \cosh \left(t-k T_{d}\right)\right]^{(2-n) / 2}} & \text { for } k \cdot T_{d}+1<t .\end{cases}
$$

The cut-and-paste processes take place in

$[-1,1]$ and $\left[k \cdot T_{d}-1, k \cdot T_{d}+1\right]$.

Using the limit in (66) and the periodicity of $w_{T_{d}}$, we find that we can make the truncated function has the corresponding scalar curvature function approaching $4 n(n-1)$ when $T_{d} \rightarrow \infty . \bar{w}_{k, T_{d}}$ can be brought back to $S^{n}$ (denoted by $\bar{U}_{k, T_{d}}$ ) via (62). Geometrically and intuitively, $k+1$ spheres are stacked up like a tower. Note that (blow-up condition)

$$
\bar{U}_{k, T_{d}}(S) \rightarrow \infty \text { as } T_{d} \rightarrow \infty
$$

and

$$
\inf _{O} \bar{U}_{k, T_{d}} \geq c^{2}>0 \text { for all } T_{d} \gg 1
$$

Here $O$ is any (non-empty) open set with $\bar{O} \subset S^{n} \backslash\{S\}$. Such a kind of blow-up is known as supported (bounded away from zero). The challenge is to "stabilize" the scalar curvature throughout.

\section{Conclusion and final remarks}

In [17], one finds challenging problems on the Monge-Ampere equation and the optimal transportation problems. For the prescribed scalar curvature, the case when $K$ equals to a positive number in a "large" region is seldom known. One can expect further exciting developments in these areas.

\section{References}

[1] K. F. Gauss. Disquisitiones generales circa superficies curvas. Societati regise oblatse D. 8. Octob, 1827.

[2] H. Minkowski. Volumen und Oberflche. Math. Ann. 1903; 57: 447-495.

[3] E. Calabi. Review of The Minkowski multidimensional problem, by Aleksey Vasilyevich Pogorelov. Bull. Amer. Math. Soc. 1979; 1: 636-639.

[4] J. Kazdan. Prescribing the Curvature of a Riemannina Manifold. AMS CBMS Lecture. 1984; 57.

[5] T. Riviére. Exploring the unknown: the work of Louis Nirenberg on partial differential equations. Notices of the AMS. 2016; 63(2): 120-125.

[6] J. P. Bourguignon, J. P. Ezin. Scalar curvature functions in a conformal class of metrics and conformal transformations. Trans. Amer. Math. Soc. 1987; 301: 723-736.

[7] L. Ambrosio, N. Gigli. A user's guide to optimal transport, CIME summer school, Italy, (hal-00769391), 2009.

[8] A. M. Alghamdi S. Gala, M. A. Ragusa. On the blow-up criterion for incompressible Stokes-MHD equations.Results in Mathematics. 2018; 73: 1-6.

[9] A. Bahri. Critical Points at Infinity in Some Variational Problems, Pitman Res. Notes Math. Longman, Harlow, U.K., $1989 ; 182$.

[10] H. Busemann. Convex Surfaces. Interscience. New York, 1958.

[11] L. Caffarelli, B. Gidas, J. Spruck. Asymptotic symmetry and local behavior of semilinear elliptic equations with critical Sobolev growth. Comm. Pure Appl. Math. 1989; 42: 271-297.

[12] A. S.-Y. Chang, P.-C. Yang. A perturbation result in prescribing scalar curvature on $S^{n}$. Duke Math. J. 1991; 64: $27-69$.

[13] C.-C. Chen, C.-S. Lin. Estimates of the conformal scalar curvature equation via the method of moving planes.Comm. Pure Appl. Math. 1997; 50: 971-1017.

[14] C.-C. Chen, C.-S. Lin. Estimate of the conformal scalar curvature equation via the method of moving planes. II, $J$. Differential Geom. 1998; 49: 115-178.

[25] X.-Z. Chen, X.-W. Xu. The scalar curvature flow on $S^{n}$-perturbation theorem revisited. Invent. Math. 2012, 187: 395506 [Erratum: Invent. Math. 2012; 187: 507-509].

[16] H. Chtioui, A. Rigane, On the prescribed Q-curvature problem on $S^{n}$. J. Func. Anal. 2011; 261: 2999-3043. 
[17] G. De Philippis, A. Figalli. The Monge-Ampére equation and its link to optimal transportation. Bull. Amer. Math. Soc. 2014; 51: 527-580.

[18] L. G. Farah, J. Holmer \& S. Roudenko. Instability of solitons-revisited, I: The critical generalized KdV equation, Conference: AMS-MAA. Contemporary Mathematics. 2019; 725: 65-88.

[19] M.-C. Leung. Conformal scalar curvature equations on complete manifolds. Comm. Partial Differential Equations. 1995; 20: 367-417.

[20] M.-C. Leung, Blow-up solutions of nonlinear elliptic equations in $I R^{n}$ with critical exponent, Math. Ann. 2003; 327: 723-744.

[21] M.-C. Leung, Refined estimates for simple Blow-ups of the scalar curvature equation on $S^{n}$. Trans. Amer. Math. Soc. 2018; 370: 1159-1184.

[22] Y.-Y. Li. Prescribing scalar curvature on $S^{n}$ and related problems. I, J. Differential Equations. 1995; 120: 319-410.

[23] Y.-Y. Li. Prescribing scalar curvature on $S^{n}$ and related problems. II. Existence and compactness. Comm. Pure Appl. Math. 1996; 49: 541-597.

[24] J. Murphy. The nonlinear Schrodinger equation with an inverse-square potential, Conference: AMS-MAA, Contemporary Mathematics. 2019; 725: 215-225.

[25] T.-H. Pak. Webster scalar curvature flow on CR sphere. I \& II, Adv. Math. 2015; 268: 758-905.

[26] C. Villani. Optimal Transport: Old and New, Springer. New Yori, 2008.

[27] A. D. Alexandrov. Existence and uniqueness of a convex surface with a given integral curvature. C. R. (Doklady) Acad. Sci. URSS (N. S.). 1942; 35: 131-134.

[28] A. D. Alexandrov. Smoothness of the convex surface of bounded Gaussian curvature. C. R. (Doklady) Acad. Sci. URSS (N. S.). 1942; 36: 195-199.

[29] A. D. Alexandrov. Dirichlets problem for the equation det $\left\|z_{i j}\right\|=\psi\left(z_{1}, \ldots, z_{n}, z, x_{1}, \ldots, x_{n}\right)$ I. (Russian) Vestnik Leningrad. Univ. Ser. Mat. Meh. Astr. 1958; 13: 5-24.

[30] A. V. Pogorelov. The regularity of the generalized solutions of the equation $\operatorname{det}\left(\partial^{2} u / \partial x^{i} \partial x^{j}\right)=\varphi\left(x_{1}, x_{2}, \ldots ., x_{n}\right)>0$. (Russian) Dokl. Akad. Nauk SSSR. 1971; 200: 534-537.

[31] L. Caffarelli. Some regularity properties of solutions of Monge-Ampére equation. Comm. Pure Appl. Math. 1991; 44: 965-969.

[32] A. Figalli. On the Monge-Ampére equation. Séminaire Bourbaki. 2017-2018; 1147.

[33] P. Guan, Y. Y. Li. C1,1 estimates for solutions of a problem of Alexandrov. Comm. Pure Appl. Math. 1997; 50: 789811.

[34] G. Monge. Mémoire sur la théorie des déblais et des remblais. Histoire de l'Acadmie Royale des Sciences de Paris, avec les Mémoires de Mathematique et de Physique pour la même année. 1781; 666-704.

[35] Y. Brenier, Polar factorization and monotone rearrangement of vector-valued functions. Comm. Pure Appl. Math. $1991 ; 44: 375-417$.

[36] X.-N. Ma. N. Trudinger, X.-J. Wan. Regularity of potential functions of the optimal transportation problem. Arch. Ration. Mech. Anal. 2005; 177: 151-183.

[37] A. Ambrosetti, A. Malchiodi. Perturbation Methods and Semilinear Elliptic Problems on $I R^{n}$. Progress in Mathematics 240. Birkhäuser, Basel-Boston-Berlin, 2006.

[38] A. Bahri, J. Coron. The scalar-curvature problem on the standard three-dimensional sphere. J. Funct. Anal. 1991; 95: 106-172.

[39] Z.-C Han, Y.-Y. Li. A note on the Kazdan-Warner type condition, Ann. Inst. H. Poincaré Anal. Non Linéaire. 1996, 13: 283-292.

[40] M.-C. Leung, F. Zhou. Prescribed scalar curvature equation on Sn in the presence of reflection or rotation symmetry. Proc. Amer. Math. Soc. 2014, 142: 1607-1619.

[41] M.-C. Leung. Construction of blow-up sequences for the prescribed scalar curvature equation on Sn. I. Uniform cancellation, Comm. Contemporary Mathematics. 2012; 14: 1-31.

[42] D. Gilbarg, N. Trudinger. Elliptic Partial Differential Equations of Second Order, second edition, Springer-Verlag, Berlin-Heidelberg-New York, 1998.

[43] J. Wei, S. Yan. Infinitely many solutions for the prescribed scalar curvature problem on $S^{N}$. J. Funct. Anal. 2010; 258: 3048-3081.

[44] M.-C. Leung, F. Zhou. Conformal scalar curvature equation on $S^{n}$ : functions with two close critical points (twin pseudo-peaks). Comm. Contemporary Mathematics. 2018; 20: 34-66.

[45] M.-C. Leung. Supported blow-up and prescribed scalar curvature on $S^{n}$. Mem. Amer. Math. Soc. 2011; $231: 1002$.

[46] S. Brendle. Blow-up phenomena for the Yamabe equation. J. Amer. Math. Soc. 2008; 21: 951-979.

[47] S. Brendle, F. Marques. Blow-up phenomena for the Yamabe equation. II, J. Differential Geom. 2009; 81: $225-250$.

[48] M. Khuri, F. Marques, R. Schoen. A compactness theorem for the Yamabe problem. J. Differential Geom. 2009; 81: 
143-196.

[49] M.-C. Leung. Construction of blow-up sequences for the prescribed scalar curvature equation on $S^{n}$. II. Annular domains, Calc. Var. Partial Differential Equations. 2013; 46: 1-29.

[50] M.-C. Leung, F. Zhou. Construction of blow-up sequences for the prescribed scalar curvature equation on $S^{n}$. III. Aggregated and towering Blow-up. Calc. Var. Partial Differential Equations. 2015; 54: 3009-3035.

[51] N. Korevaar, R. Mazzeo, F. Pacard, R. Schoen. Refined asymptotics for constant scalar curvature metrics with isolated singularities. Invent. Math. 1999; 135: 233-272.

[52] M.-C. Leung. Exotic solutions of the conformal scalar curvature equation in $I R^{n}$. Annales de l'Institut Henri PoincareAnalyse Non Lineaire. 2001; 18: 297-307.

[53] R. Mazzeo, F. Pacard. Constant scalar curvature metrics with isolated singularities. Duke Math. J. 1999; 99: 353-418. 